\section{Dr. Sugawara et al reply}

\section{To the Editor:}

We sincerely thank Dr. Wang et al for their comments in response to our article, "Cytomegalovirus Enteritis in a Patient with Rheumatoid Arthritis Receiving Baricitinib."' Our case report had demonstrated increased risk of cytomegalovirus (CMV) infection under the combination therapy of baricitinib and tacrolimus.

As Dr. Wang et al pointed out, ${ }^{2}$ this case report is extremely brief, and minimal clinical information was included, because it was submitted following the requirement of an "Images in Rheumatology" article of The Journal of Rheumatology. We also agree that detailed information of the patient will be helpful for understanding this case report. We herein describe the patient in detail based on our original case report. ${ }^{1}$ This study was reviewed and approved by the Ethics Committee of Tonan Hospital (approval no. 434); the patient's written informed consent was obtained.

In 2004, a 65-year-old male was diagnosed with rheumatoid arthritis (RA) based on the 1987 American College of Rheumatology criteria for classification of $\mathrm{RA}^{3}$. He was negative for either anticyclic citrullinated peptide antibodies or rheumatoid factor. He had no history of radiation therapy. He received $12 \mathrm{mg} /$ week of methotrexate (MTX), $6 \mathrm{mg} / \mathrm{kg}$ of infliximab (IFX) at 8 -week intervals, $2 \mathrm{mg} /$ day of tacrolimus, and low-dose prednisolone, and was in remission for 13 years. Due to secondary failure of his RA, IFX was stopped in 2018 and $4 \mathrm{mg}$ of baricitinib was started. After administration of baricitinib, he achieved clinical remission. Six months after starting baricitinib, the patient experienced CMV enteritis as described in our case report. ${ }^{1}$

As Dr. Wang et al commented, ${ }^{2}$ we have to be careful about gastrointestinal infections. Second, our patient did not have high $\mathrm{C}$-reactive protein (CRP) levels, suggesting that the patient might have good prognosis. However, Janus kinase (JAK) and signal transducers and activators of transcription (STAT) signaling pathway mediates cellular responses to interleukin 6 , which regulates acute-phase proteins including CRP. Baricitinib selectively inhibits JAK $1 / 2,{ }^{4}$ leading to suppression of CRP that may result in underestimation of the severity of infection. Therefore, we must pay special attention to patients treated with baricitinib even if they do not have high CRP levels. Finally, this patient had no history of radiation therapy. In patients with a history of radiation, the dysfunction of local mucosal lymph tissue may coexist; therefore, we have to estimate these risk factors carefully. As described above, this patient was also treated with low-dose prednisolone. As Dr. Wang et al pointed out, ${ }^{2}$ we cannot exclude the immunosuppressive effect of prednisolone. It is important to try to lower the amount of prednisolone in patients with immunosuppressive therapy.

We again thank Dr. Wang et $\mathrm{al}^{2}$ for giving us an opportunity to discuss our case report. We believe that this case report will alert rheumatologists to CMV enteritis in patients with RA treated with JAK inhibitors including baricitinib, in combination with tacrolimus.

Eri Sugawara ${ }^{1}$ (D), MD, PhD

Kazuo Matsui ${ }^{1}, \mathrm{MD}$

Yoshiharu Amasaki ${ }^{1}, \mathrm{MD}, \mathrm{PhD}$

${ }^{1}$ Department of Rheumatology, Tonan Hospital, Sapporo, Japan.

The authors declare no conflicts of interest relevant to this article.

Address correspondence to Dr. E. Sugawara, 060-0004 N4W7, Chuo-Ku, Sapporo, Japan.Email: e.sugawara@huhp.hokudai.ac.jp.

\section{REFERENCES}

1. Sugawara E, Matsui K, Amasaki Y. Cytomegalovirus enteritis in a patient with rheumatoid arthritis receiving baricitinib. J Rheumatol 2020;47:1835-6.

2. Wang G, Zhuo N, Li J. Cytomegalovirus enteritis induced by baricitinib and tacrolimus: association or coincidence? J Rheumatol xxxxxxxx.

3. Arnett FC, Edworthy SM, Bloch DA, et al. The American Rheumatism Association 1987 revised criteria for the classification of rheumatoid arthritis. Arthritis Rheum 1988;31:315-24.

4. Fridman JS, Scherle PA, Collins R, et al. Selective inhibition of JAK1 and JAK2 is efficacious in rodent models of arthritis: preclinical characterization of Incb028050. J Immunol 2010;184:5298-307. 